Article

\title{
Optimal Consumption and Investment with Labor Income and European/American Capital Guarantee
}

\section{Morten Tolver Kronborg}

ATP (Danish Labour Market Supplementary Pension Scheme), Kongens Vænge 8, 3400 Hillerød, Denmark, and, Department of Mathematical Sciences, University of Copenhagen, Universitetsparken 5, 2100 Copenhagen Ø, Denmark; E-Mail: mtk@atp.dk; Tel.: +45-48-204-537.

Received: 10 March 2014; in revised form: 5 May 2014 / Accepted: 7 May 2014 /

Published: 16 May 2014

\begin{abstract}
We present the optimal consumption and investment strategy for an investor, endowed with labor income, searching to maximize utility from consumption and terminal wealth when facing a binding capital constraint of a European (constraint on terminal wealth) or an American (constraint on the wealth process) type. In both cases, the optimal strategy is proven to be of the option-based portfolio insurance type. The optimal strategy combines a long position in the optimal unrestricted allocation with a put option. In the American case, where the investor is restricted to fulfill a capital guarantee at every intermediate time point over the interval of optimization, we prove that the investor optimally changes his budget constraint for the unrestricted allocation whenever the constraint is active. The strategy is explained in a step-by-step manner, and numerical illustrations are presented in order to support intuition and to compare the restricted optimal strategy with the unrestricted optimal counterpart.
\end{abstract}

Keywords: stochastic control; martingale method; option-based portfolio insurance; American put option; human capital; borrowing constraint; CRRA utility

\section{Introduction}

We study the optimal consumption and investment decision problem for an investor, endowed with deterministic labor income, who faces an American capital guarantee restriction. An American capital guarantee is a capital constraint that is valid at all time points in the time interval of consideration. Likewise, we refer to a capital guarantee only valid at the terminal time point as a European capital 
guarantee. The names are inherited from the option framework, i.e., from American and European options. The problem is set in a continuous-time frictionless Black-Scholes market, and the investor measures his tolerance towards risk by a constant relative risk aversion utility function.

The corresponding free consumption and investment decision problem, where the investor is not restricted to fulfill any capital guarantee, is a well-understood problem; see Merton [1] and Merton [2]. Traditionally, such problems have been solved using stochastic dynamic programming. However, stochastic dynamic programming becomes difficult when adding a capital constraint, since the value function, in addition to a stochastic differential equation, must satisfy some boundary condition whenever the wealth is at the boundary. This leaves us with a non-linear Hamilton-Jacobi-Bellman equation for which the existence of a solution has to be established.

Consequently, this article uses, instead, the martingale methodology developed by Karatzas et al. [3], Cox and Huang [4] and Cox and Huang [5]. We adopt the ideas from El-Karoui et al. [6] and use the put option-based approach to derive the optimal consumption and investment strategy for an investor who faces an American capital guarantee restriction defined as a deterministic function. It turns out that the optimal strategy consists of investing a part of the initial total wealth ${ }^{1}$ in the corresponding optimal unrestricted allocation and the remainder in a put option written on the optimal unrestricted portfolio. In order for this strategy to be admissible, we show that the investor must adjust the portfolio continuously whenever the constraint is active. This result is similar to the one of El-Karoui et al. [6], although they do not allow for consumption and labor income. The main contribution of this article is that we show that the put option-based approach can also be carried out to find the optimal strategy in the case of including consumption and labor income. Clearly, the introduction of consumption leads to further mathematical difficulties; nor is the introduction of deterministic labor income trivial. One important thing to note is, in contrast to the unrestricted problem of Merton, being endowed with labor income or being endowed with an initial wealth enlarged by the financial value of future labor income is not equivalent when considering an American capital guarantee restriction. In the case of an American capital guarantee, the latter case is preferable. This indicates that adding deterministic labor income to the setting considered in El-Karoui et al. [6] is not as easy as it may seem. The strategy actually differs from the case excluding deterministic labor income. We show that adding deterministic labor income can be handled by buying an insurance (a put option) with a strike price substantially different from the one obtained by El-Karoui et al. [6]. To ease the understanding of the optimal strategy derived, the analysis also contains a step-by-step explanation of the optimal strategy together with a numeric graph-based explanation. In the special case of a restriction to borrow against future labor income (an American capital guarantee equal to zero), we compare numerically the restricted solution with the unrestricted counterpart.

A reasonable question is whether a wealth constraint is relevant for an individual endowed with deterministic labor income. As mentioned, for the unrestricted problem, the investor optimally sells his future labor income in the market and acts as if he had an enlarged initial wealth. Consequently, the investor's terminal wealth becomes positive, but the investor's wealth may become negative at some

1 Initial wealth plus the financial value of future labor income. 
point in time before the terminal time. If labor income really is deterministic, one could argue that this is perfectly acceptable. The motivation of generally introducing an American capital guarantee, and specifically, to analyze the case of a restriction to borrow against future labor income, is, however, manifold. Consider, for example, the lending business. In reality, you can borrow a great amount of money from the bank to buy a house if you are the receiver of a (possible stochastic) labor income. The reason is that you invest your loan in the house and, thereby, maintain a positive wealth. Of course, the value of the house could drop, but since the loan comes with an amortization plan, ${ }^{2}$ the wealth stays positive (with a high probability). You can also get a mortgage loan from the bank to finance consumption. On the other hand, it is almost impossible to get a loan from the bank to finance pure consumption if you cannot provide security for the loan. ${ }^{3}$ Why is that? Of course, this is partly because the bank considers your labor income stochastic (e.g., you might lose your job), but there are many other reasons. Most of them are connected to obvious reasons, such as moral hazards, adverse selection and administration problems. Or one could say that the bank just does not want to take on that risk. This paper is motivated by the real life situation that many individuals face. As an individual, you do not consider your labor income as stochastic. At least, you do not act as if you do. This is partly because it is very hard to model labor income, since the list of relevant stochastic variables to include in the modulation would be very long. The modeling should, among other things, include future political decisions, possible world wars, personal accidents (you may become disabled), your mortality (you may die) and the demand of tomatoes (if you make a living off selling tomatoes). In addition, many people actually have a very stable and secure labor income and, thereby, have a very strong belief about what their life-long labor income would be. If this were not true, why are more people not buying unemployment insurance? Summing up, the question many individuals face is: you consider your labor income deterministic, and you act responsibly as a good citizen. Unfortunately, the bank does not agree with you, and consequently, the bank is only willing to issue a loan if you invest in such a way that they do not stand the chance of ending up with a loss (your total assets must be positive at all times). Given this situation, how should you optimally consume and invest? This is one interpretation of the question considered in this article.

Furthermore, the problem studied in this article is putting consumption equal to zero; highly relevant for many financial institutions. The most obvious example is pension companies, which are forced by law (by the Financial Supervisory Authority (FSA)) to fulfill certain capital requirements at all times. In such a framework, labor income has the interpretation as pension contributions, and the American capital guarantee, introduced by the FSA, puts restrictions on the pension company's actions.

The paper by Lakner and Nygren [7] considers a similar problem. They maximize expected utility from consumption and terminal wealth when both the consumption rate and terminal wealth must not fall below a given minimum consumption level and a minimum wealth level, respectively. This is handled by dividing initial wealth into two parts, $x_{1}$ and $x_{2}$, and then solving the constrained pure consumption problem with initial wealth $x_{1}$ and the constrained pure portfolio problem with initial wealth $x_{2}$. The

2 In addition, most banks require the investor himself to provide (say) five percent funding.

3 In some countries, this is actually possible, but since expenses are incredible high, we assume that the investor does not consider this as an opportunity. 
superposition of the actions for the two constrained subproblems is then shown to be the optimal strategy provided that $x_{1}$ and $x_{2}$ are chosen, such that the "marginal expected utilities" from the two constrained subproblems are identical. In this article, we do not consider a minimum level of consumption constraint, since we do not want the consequence of the introduction of an American capital guarantee to be blurred by other constraints. One could, using the results obtained in this article, apply the approach of Lakner and Nygren [7] to solve such a problem.

Using different approaches, the restriction to borrow against future labor income has been analyzed in different settings with a spanned stochastic income rate. He and Pages [8] use duality theory to show the existence of an optimal strategy and find that the presence of a liquidity constraint has a smoothing effect on the optimal consumption. El-Karoui and Jeanblanc [9] introduce the concept of super strategies to show that the fair-value, defined as the minimal investment required to replicate a consumption investment strategy with a super strategy, consists of investing a part of the initial wealth in a free portfolio and to use the remaining part to buy an American put option on this underlying asset. A setting with unspanned stochastic income is analyzed by Duffie and Zariphopoulou [10], Duffie et al. [11], Koo [12] and Munk [13], who also give a very complete overview of the field as a whole.

The paper is organized as follows: In Section 2, we present the economic model, the concept of admissible strategies and other preliminaries. In Section 3, we solve the unrestricted problem using the martingale technique. As an inspirational example, we solve, in Section 4, the optimal consumption and investment decision problem when the investor must fulfill a binding terminal capital guarantee (any terminal guarantee strictly greater than zero), also known as a European capital guarantee. Using the building blocks derived in Sections 3 and 4, and the theory of American put options in a Black-Scholes market, we construct, in the main section, Section 5, an admissible put option-based portfolio insurance strategy. We show that this strategy is optimal for the consumption and investment decision problem, where the investor must fulfill an American capital guarantee. Finally, in Subsection 5.3, numerical illustrations of the optimal restricted strategy together with a comparison with the optimal unrestricted strategy are presented for the special case of a restriction to borrow against future labor income.

\section{Setup}

Consider a frictionless Black-Scholes market consisting of a bank account, $B$, with risk-free short rate, $r$, and a risky stock, $S$, with dynamics given by:

$$
\begin{aligned}
& d B(t)=r B(t) d t, B(0)=1 \\
& d S(t)=\alpha S(t) d t+\sigma S(t) d W(t), S(0)=s_{0}>0
\end{aligned}
$$

Here, $\alpha, \sigma, r>0$ are constants, and it is assumed that $\alpha>r$. The process, $W$, is a standard Brownian motion on an abstract probability space $(\Omega, \mathcal{F}, P)$ equipped with the filtration $\mathbb{F}^{W}=\left(\mathcal{F}^{W}(t)\right)_{t \in[0, T]}$ given by the $P$-augmentation of the filtration $\sigma\{W(s) ; 0 \leq s \leq t\}, \forall t \in[0, T]$.

We consider an investor endowed with a continuous deterministic labor income of rate $\ell$, an initial amount of money, $x_{0}$, and a time horizon of interest, $[0, T], T>0$. Over the time interval, $[0, T]$, the investor has the opportunity to continuously consume a fraction of his wealth and to invest his wealth in the Black-Scholes market. More specifically, let $c$ denote the rate of consumption, and let $\pi$ denote the 
fraction of wealth to be invested in the risky asset, $S$. For a given strategy, $(c, \pi)$, the wealth process of the investor, $(X(t))_{t \in[0, T]}$, is given by the dynamics:

$$
\begin{aligned}
d X(t) & =[(r+\pi(t)(\alpha-r)) X(t)+\ell(t)-c(t)] d t+\sigma \pi(t) X(t) d W(t), t \in[0, T) \\
X(0) & =x_{0} \geq 0
\end{aligned}
$$

We assume the consumption rate, $c(t), t \in[0, T]$, to be a non-negative, $\mathcal{F}^{W}(t)$-adapted process with $\int_{0}^{T} c(t) d t<\infty$ a.s., and the investment strategy, $\pi(t), t \in[0, T]$, to be an $\mathcal{F}^{W}(t)$-adapted process, such that (1) has a unique solution, $X(t), t \in[0, T]$, satisfying $\int_{0}^{T}(\pi(t) X(t))^{2} d t<\infty$ a.s. We have, $\forall t \in[0, T]$, the representation:

$$
X(t)=x_{0} e^{r t}+\int_{0}^{t} e^{r(t-s)}[\pi(s) X(s)(\alpha-r)+\ell(s)-c(s)] d s+\int_{0}^{t} e^{r(t-s)} \sigma \pi(s) X(s) d W(s)
$$

It is well known that in the Black-Scholes market, the equivalent martingale measure, $Q$, is unique and given by the Radon-Nikodym derivative:

$$
\frac{d Q(t)}{d P(t)}=\Lambda(t):=\exp \left(-\left(\frac{\alpha-r}{\sigma}\right) W(t)-\frac{1}{2}\left(\frac{\alpha-r}{\sigma}\right)^{2} t\right), t \in[0, T]
$$

and that the process, $W^{Q}$, given by $W^{Q}(t)=W(t)+\frac{\alpha-r}{\sigma} t, t \in[0, T]$, is a standard Brownian motion under $Q$. We get that the investor's wealth can be represented, $\forall t \in[0, T]$, by:

$$
X(t)=x_{0} e^{r t}+\int_{0}^{t} e^{r(t-s)}(\ell(s)-c(s)) d s+\int_{0}^{t} e^{r(t-s)} \sigma \pi(s) X(s) d W^{Q}(s)
$$

Definition 1. Define the set of admissible strategies, denoted by $\mathcal{A}$, as the consumption and investment strategies for which the corresponding wealth process given by (2) is well-defined,

$$
X(t)+g(t) \geq 0, \forall t \in[0, T]
$$

where $g$ is the time, $t$, financial value of future labor income defined by $g(t):=\int_{t}^{T} e^{-r(s-t)} \ell(s) d s$, and:

$$
E^{Q}\left[\int_{0}^{T} e^{-r s} \sigma \pi(s) X(s) d W^{Q}(s)\right]=0
$$

The technical condition (4) is equivalent to the condition that under $Q$, the process:

$$
\int_{0}^{t} e^{-r s} \sigma \pi(s) X(s) d W^{Q}(s), t \in[0, T]
$$

is a martingale (in general, it is only a local martingale and also a supermartingale if (3) is fulfilled; see, e.g., Karatzas and Shreve [14]). From this, we conclude that (4) insures that $(c, \pi) \in \mathcal{A}$, if and only if $X(T) \geq 0$ and, $\forall t \in[0, T]$,

$$
X(t)+g(t)=E^{Q}\left[\int_{t}^{T} e^{-r(s-t)} c(s) d s+e^{-r(T-t)} X(T) \mid \mathcal{F}^{W}(t)\right]
$$

At time zero, this means the strategies have to fulfill the budget constraint:

$$
x_{0}+g(0)=E^{Q}\left[\int_{0}^{T} e^{-r t} c(t) d t+e^{-r T} X(T)\right]
$$

For latter use, we state the following remark. 
Remark 1. Define

$$
Z(t):=\int_{0}^{t} e^{-r s}(c(s)-\ell(s)) d s+e^{-r t} X(t), t \in[0, T]
$$

By (2), we have that Condition (4) is fulfilled, if and only if $Z$ is a martingale under $Q$. The natural interpretation is that under $Q$, the discounted wealth plus discounted consumption excess of labor income should be a martingale. It is useful to note that if the dynamics of $X$ can be represented in the form:

$$
d X(t)=\left[(r X(t)+\ell(t)-c(t)] d t+\phi(t) d W^{Q}(t), t \in(0, T]\right.
$$

for some $\mathcal{F}^{W}(t)$-adapted process, $\phi$, satisfying $\phi(t) \in £^{2}, \forall t \in[0, T]$, then $Z$ is a martingale under $Q$.

Finally, by Condition (3), we allow the wealth process to become negative, as long as it does not exceed (in absolute value) the financial value of future labor income. Note that Condition (3) puts a lower boundary on the wealth process and, therefore, rules out doubling strategies (see, e.g., Karatzas and Shreve [14]).

\section{The Unrestricted Control Problem}

For the rest of this paper, we assume that the investor's preferences can be stated in terms of a constant relative risk aversion (CRRA) utility function $u:[0, \infty) \rightarrow[-\infty, \infty)$ in the form:

$$
u(x)= \begin{cases}\frac{x^{\gamma}}{\gamma}, & \text { if } x>0 \\ \lim _{x \searrow 0} \frac{x^{\gamma}}{\gamma}, & \text { if } x=0\end{cases}
$$

for some $\gamma \in(-\infty, 1) \backslash\{0\}$. In the unrestricted control problem, known as Merton's problem (Merton [1] and Merton [2]), the investor seeks to find the strategy fulfilling the supremum:

$$
\sup _{(c, \pi) \in \mathcal{A}^{\prime}} E\left[\int_{0}^{T} e^{-\int_{0}^{t} \beta(s) d s} u(c(t)) d t+e^{-\int_{0}^{T} \beta(s) d s} u(X(T))\right]
$$

where $X(0)=x_{0}$ and $\beta$ is a deterministic function representing the investor's time preferences. The supremum in (8) is taken over the subset of the admissible strategies, referred to as the feasible strategies, defined by:

$$
\mathcal{A}^{\prime}:=\left\{(c, \pi) \in \mathcal{A} \mid E\left[\int_{0}^{T} e^{-\int_{0}^{t} \beta(s) d s} \min (0, u(c(t))) d t+e^{-\int_{0}^{T} \beta(s) d s} \min (0, u(X(T)))\right]>-\infty\right\}
$$

i.e., it is allowed to draw an infinite utility from a strategy, $(c, \pi) \in \mathcal{A}^{\prime}$, but only if the expectation over the negative parts of the utility function is finite. Clearly, for $\gamma \in(0,1)$, we have that $A$ and $A^{\prime}$ coincide.

The unrestricted control problem (8) can be solved using either the conventional Hamilton-Jacobi-Bellman (HJB) method (see, e.g., Fleming and Richel [15]) or the newer martingale method (see, Karatzas et al. [3], Cox and Huang [4] and Cox and Huang [5]). Despite the fact that this is most easily done using the HJB method, we now sketch the solution to (8) in terms of the martingale 
method. We do so because the solution to the corresponding American capital guarantee version of (8), presented in Section 5, is based on terms derived from the martingale method.

Define the inverse of the derivative of $u$ as the function $I:(0, \infty] \rightarrow[0, \infty)$, and define the adjusted state price deflator

$$
H(t):=\Lambda(t) e^{\int_{0}^{t}(\beta(s)-r) d s} .
$$

Since $I$ is continuous and decreasing and maps $(0, \infty]$ onto $[0, \infty)$, we get the useful result that there exists a unique constant $\xi^{*}>0$, such that (see, e.g., Karatzas and Shreve [14]):

$$
E^{Q}\left[\int_{0}^{T} e^{-r t} I\left(\xi^{*} H(t)\right) d t+e^{-r T} I\left(\xi^{*} H(T)\right)\right]=x_{0}+g(0)
$$

Finally, note that from the concavity of $u$ and $u^{\prime}(I(z))=z$, we get:

$$
u(x) \leq u(I(z))-z(I(z)-x), \forall x \geq 0, z>0
$$

Now, take an arbitrary strategy, $(c, \pi) \in \mathcal{A}^{\prime}$, with corresponding wealth process, $(X(t))_{t \in[0, T]}$, as given. Using (11), the budget constraint (5) and finally (10), we get that:

$$
\begin{aligned}
& E\left[\int_{0}^{T} e^{-\int_{0}^{t} \beta(s) d s} u(c(t)) d t+e^{-\int_{0}^{T} \beta(s) d s} u(X(T))\right] \\
\leq & E\left[\int_{0}^{T} e^{-\int_{0}^{t} \beta(s) d s} u\left(I\left(\xi^{*} H(t)\right)\right) d t+e^{-\int_{0}^{T} \beta(s) d s} u\left(I\left(\xi^{*} H(T)\right)\right)\right]
\end{aligned}
$$

Since $(c, \pi)$ was arbitrarily chosen, we conclude that the candidate optimal strategy $\left(c^{*}, \pi^{*}\right)$ is given by:

$$
\begin{aligned}
c^{*}(t) & =I\left(\xi^{*} H(t)\right) \\
X^{*}(T) & =I\left(\xi^{*} H(T)\right)
\end{aligned}
$$

where $X^{*}(T)$ is the corresponding terminal wealth obtained from using the strategy $\left(c^{*}, \pi^{*}\right)$. By use of the martingale representation theorem (see e.g., Karatzas and Shreve [16]) we obtain (after some rather cumbersome calculations):

$$
\begin{aligned}
c^{*}(t) & =\frac{X(t)+g(t)}{f(t)} \\
\pi^{*}(t) X^{*}(t) & =\frac{1}{\gamma} \frac{\alpha-r}{\sigma^{2}}\left(X^{*}(t)+g(t)\right)
\end{aligned}
$$

where:

$$
f(t)=\int_{t}^{T} e^{-\int_{t}^{s}[\widetilde{r}(y)+\beta(y)] d y} d s+e^{-\int_{t}^{T}[\widetilde{r}(y)+\beta(y)] d y}
$$

with $\widetilde{r}(y)=\frac{\gamma}{1-\gamma} \beta(y)-\frac{\gamma}{1-\gamma} r-\frac{1}{2} \frac{\gamma}{(1-\gamma)^{2}}\left(\frac{\alpha-r}{\sigma}\right)^{2}$. Inserting the candidate optimal strategy $\left(c^{*}, \pi^{*}\right)$ in (1), we observe that $X^{*}(t)+g(t), t \in[0, T]$, referred to as the total wealth, follows a time inhomogeneous geometric Brownian motion. ${ }^{4}$ More specifically, we get, $\forall t \in[0, T]$, that:

$$
X^{*}(t)+g(t)=\left(x_{0}+g(0)\right) \exp \left\{\left(r+\frac{\frac{1}{2}-\gamma}{(1-\gamma)^{2}}\left(\frac{\alpha-r}{\sigma}\right)^{2}\right) t-\int_{0}^{t} \frac{1}{f(s)} d s+\frac{1}{\gamma} \frac{\alpha-r}{\sigma} W(t)\right\}
$$

4 In particular, $X^{*}(t)+g(t), t \in[0, T]$, is a geometric Brownian motion if $\beta$ is constant. 
In particular, since $f$ is bounded away from zero, $\forall t \in[0, T]$, we have that $X^{*}(t), t \in[0, T]$, is well-defined, and clearly, (3) is fulfilled. From (14), we also conclude that the candidate optimal consumption rate is non-negative. Moreover, since $X^{*}(t)+g(t)$ is log-normally distributed, $\forall t \in[0, T]$, one can easily show that:

$$
E^{Q}\left[\int_{0}^{T}\left(\sigma \pi^{*}(t) X^{*}(t)\right)^{2} d t\right]<\infty
$$

which ensures that (4) is fulfilled. In fact, by use of the martingale representation theorem, we have that the candidate optimal investment strategy can be written as:

$$
\pi^{*}(t) X^{*}(t)=\frac{e^{r t} \psi(t)}{\sigma}, t \in[0, T]
$$

where $\psi$ is the unique integrand from the martingale representation theorem, i.e.,

$$
\int_{0}^{t} \psi(s) d W^{Q}(s), t \in[0, T]
$$

is a martingale under $Q$. From this, it follows immediately that (4) is fulfilled. Thus, the candidate optimal strategy $\left(c^{*}, \pi^{*}\right)$ is admissible. Finally, since $x_{0}$ and $g(0)$ cannot both be zero, we get, from (14) and (16), that $c^{*}(t)>0, \forall t \in[0, T]$, and $X^{*}(T)>0$, and we note that the additional condition given in (9) is fulfilled, i.e., $\left(c^{*}, \pi^{*}\right)$ is feasible. We conclude that $\left(c^{*}, \pi^{*}\right)$, given by (14) and (15), is the optimal strategy for the unrestricted problem (8).

Remark 2. It is well known that the control problem (8) with and without deterministic labor income coincides in the sense that an investor with an initial wealth equal to $x_{0}+g(0)$, but no labor income, optimally consumes and invests the same amount of money as the investor we consider. Define $Y(t):=X(t)+g(t)$ and the obvious notation $Y^{*}(t):=X^{*}(t)+g(t), \forall t \in[0, T]$. In Sections 4 and 5, we exploit the fact that an investor with wealth process $(Y(t))_{t \in[0, T]}$, i.e., no labor income, starting with an initial amount of money $y_{0}:=x_{0}+g(0)$, can replicate the wealth process, $\left(Y^{*}(t)\right)_{t \in[0, T]}$, by following the consumption and investment strategy given, $\forall t \in[0, T]$, by:

$$
\begin{aligned}
c^{*}(t) & =\frac{Y^{*}(t)}{f(t)} \\
\pi_{y}^{*} & =\frac{1}{\gamma} \frac{\alpha-r}{\sigma^{2}}
\end{aligned}
$$

From (16), it follows that $Y^{*}(t), \forall t \in[0, T]$, is proportional to the initial amount of money, $y_{0}$. We see that starting out with the initial amount of money, $\lambda y_{0}, \lambda \in \mathbb{R}_{+}$, and no labor income, one can replicate $\lambda Y^{*}(t), \forall t \in[0, T]$, by following the strategy $\left(\lambda c^{*}, \pi_{y}^{*}\right)$.

Note that the optimal strategy $\left(c^{*}, \pi^{*}\right)$ guarantees that $X^{*}(t)>-g(t), \forall t \in[0, T]$. This is in some sense a direct consequence of the CRRA utility. Loosely speaking, the marginal increase in utility is going to infinity as terminal wealth is going to zero, i.e., a terminal wealth equal to zero cannot be optimal. To be able to avoid this in all scenarios, the investor simply ensures that $X^{*}(t)>-g(t), \forall t \in[0, T]$. As mentioned in Section 1, one should also notice that the formulation 
of Problem (8) assumes that the bank allows the investor to have a negative wealth at any given time point, $t \in(0, T)$. This will, in reality, due to reasons discussed in the Introduction, likely not be the case. Furthermore, most investors tend to have some sort of preference or demand for a capital guarantee. The most obvious example is that pension companies often are required by authorities (FSA) to fulfill a certain American capital guarantee. Jensen and Sorensen [17] quantify that an individual facing a (European) mandatory minimum interest rate guarantee, as an integrated part of the pension contract, is forced to invest in an embedded put option. They argue that the investor may face a constraint on the feasible set of portfolio choices, which may induce a significant utility loss.

Including an American capital guarantee with Problem (8) is the main problem of interest in this article. Inspired by El-Karoui et al. [6], we, as an inspirational example, start out by solving the easier case, including a binding European capital guarantee. This problem was first solved by Tepla [18].

\section{The European Capital Guarantee Control Problem}

Consider the problem:

$$
\sup _{(c, \pi) \in \mathcal{A}^{\prime}} E\left[\int_{0}^{T} e^{-\int_{0}^{t} \beta(s) d s} u(c(t)) d t+e^{-\int_{0}^{T} \beta(s) d s} u(X(T))\right]
$$

under the capital constraint $X(T) \geq K$. In Section 3, we saw that $K \leq 0$ is a non-binding constraint. Note that in order for the European capital guarantee problem to be solvable and non-trivial ${ }^{5}$, we must demand that:

$$
K<e^{r T}\left(x_{0}+g(0)\right)
$$

Proposition 2 presents the optimal strategy for Problem (20). The strategy is based on a so-called option-based portfolio insurance (OBPI) written on a 'budget-adjusted' version of the optimal portfolio derived for the unrestricted problem. First, we need some notation: denote by $P_{X}^{e}(t, T, K)$ the time, $t$, value of a European put option with strike price $K$ and maturity $T$ written on a portfolio, $(X(s))_{s \in[t, T]}$. As in Section $3, X^{*}(t), c^{*}(t)$ and $\pi^{*}(t), t \in[0, T]$, denote the optimal wealth, the optimal consumption strategy and the optimal investment strategy for the unrestricted problem (8), respectively.

Proposition 2. Consider the strategy $\left(\widehat{c}, \lambda Y^{*}\right)$, where, $\forall t \in[0, T]$,

$$
\begin{aligned}
\widehat{c}(t) & :=\frac{\lambda Y^{*}(t)}{f(t)}=\lambda c^{*}(t) \\
Y^{*}(t) & :=X^{*}(t)+g(t)
\end{aligned}
$$

combined with a position in a European put option with strike price $K$ and maturity $T$ written on the portfolio, $\left(\lambda Y^{*}(s)\right)_{s \in[t, T]}$, where $\lambda$ is determined by the budget constraint:

$$
\widehat{X}^{(\lambda)}(0):=\lambda\left(x_{0}+g(0)\right)+P_{\lambda Y^{*}}^{e}(0, T, K)-g(0)=x_{0}
$$

5 In the case of equality in (21), the investor has no choice, but to invest all his wealth, including labor income, in the risk-free short rate to be sure to fulfil the capital guarantee. 
Observing that $Y^{*}(T)=X^{*}(T)$, it follows that the terminal value of the corresponding option-based portfolio insurance becomes:

$$
\widehat{X}^{(\lambda)}(T):=\lambda X^{*}(T)+\left(K-\lambda X^{*}(T)\right)^{+}=\max \left(\lambda X^{*}(T), K\right)
$$

i.e., the European capital guarantee is fulfilled. We have that:

(1) The strategy is affordable, i.e., there exist a unique $\lambda \in(0,1)$, such that the budget constraint (22) is fulfilled.

(2) The strategy is optimal for the European capital guarantee control problem given by (20).

Note that, clearly, the strategy in Proposition 2 is feasible, since the unrestricted strategy $\left(c^{*}, \pi^{*}\right)$ given in Section 3 is feasible.

Remark 3. To clarify, following the strategy, $\left(\widehat{c}, \widehat{X}^{(\lambda)}\right)$, given by Proposition 2 means that the investor should:

(1) Take a loan of size $g(0)$ and reserve the labor income to pay back the loan over the time interval $[0, T]$.

(2) Reserve the initial amount of money, $\lambda\left(x_{0}+g(0)\right)$, to follow the consumption and investment strategy $(\widehat{c}, \pi)$ given by:

$$
\begin{aligned}
\widehat{c}(t) & :=\lambda c^{*}(t) \\
\pi & :=\pi_{y}^{*}
\end{aligned}
$$

Doing this, the investor replicates the portfolio, $\lambda Y^{*}(t), \forall t \in[0, T]$, i.e., the investor replicates the terminal value $\lambda Y^{*}(T)=\lambda X^{*}(T)$ (see Remark 2).

(3) Use the remaining initial amount of money, $(1-\lambda)\left(x_{0}+g(0)\right)$, to buy a European put option with strike price $K$ and time to maturity $T$ written on the portfolio, $\left(\lambda Y^{*}(t)\right)_{t \in[0, T]}$.

The European put option is likely not to be sold in the market, but since the market is complete and frictionless, such options can be replicated dynamically by a Delta-hedge, i.e., the optimal investment strategy, $\widehat{\pi}(t), t \in[0, T]$, is given by:

$$
\widehat{\pi}(t) \widehat{X}^{(\lambda)}(t)=\left(1+\frac{\partial}{\partial y} P_{\lambda Y^{*}}^{e}(t, T, K)\right) \pi_{y}^{*} \lambda Y^{*}(t)
$$

Since the European put option has the opposite exposure to changes in the underlying portfolio, $\lambda Y^{*}(t), t \in[0, T]$, we note that the total amount of money to invest in the risky asset, $S$, becomes smaller when we introduce a European capital guarantee to the control problem (8).

Proof of affordability. First note that $\widehat{X}^{(\lambda)}(T)=\max \left(\lambda X^{*}(T), K\right)$ is non-decreasing in $\lambda$ with values in $[K, \infty)$. For $\lambda_{1}<\lambda_{2}$, we get:

$$
0 \leq \widehat{X}^{\left(\lambda_{2}\right)}(T)-\widehat{X}^{\left(\lambda_{1}\right)}(T) \leq\left(\lambda_{2}-\lambda_{1}\right) X^{*}(T)
$$


Discounting and taking the expectation under $Q$, we get, by use of (5), that:

$$
\begin{aligned}
0 & \leq \widehat{X}^{\left(\lambda_{2}\right)}(0)-\widehat{X}^{\left(\lambda_{1}\right)}(0) \leq\left(\lambda_{2}-\lambda_{1}\right) E^{Q}\left[e^{-r T} X^{*}(T)\right] \\
& =\left(\lambda_{2}-\lambda_{1}\right)\left(x_{0}+g(0)-E^{Q}\left[\int_{0}^{T} e^{-r t} c^{*}(t) d t\right]\right) \\
& <\left(\lambda_{2}-\lambda_{1}\right)\left(x_{0}+g(0)\right)
\end{aligned}
$$

We conclude that $\widehat{X}^{(\lambda)}(0)$ is an increasing and Lipschitz function with respect to $\lambda$ (Lipschitz constant equal to $\left.x_{0}+g(0)\right)$. No-arbitrage gives us $\widehat{X}^{(\lambda)}(0) \in\left[e^{-r T} K-g(0), \infty\right)$. Since $K<e^{r T}\left(x_{0}+g(0)\right)$ and $\widehat{X}^{(1)}(0)>x_{0}$, we conclude that there exists a unique $\lambda \in(0,1)$ fulfilling (22).

Before we prove the optimality of the strategy in Proposition (2), we present the following well-known result (see, e.g., Karatzas and Shreve [14]):

Lemma 3. Since $\left(c^{*}, X^{*}\right)$ is the optimal strategy for the unrestricted problem (8), we have that:

$$
\begin{aligned}
& \frac{\partial}{\partial \epsilon} E\left[\int_{0}^{T} e^{-\int_{0}^{t} \beta(s) d s} u\left(\epsilon c^{*}(t)+(1-\epsilon) c(t)\right) d t+e^{-\int_{0}^{T} \beta(s) d s} u\left(\epsilon X^{*}(T)+(1-\epsilon) X(T)\right)\right]_{\mid \epsilon=1}=0 \\
& \Longrightarrow E\left[\int_{0}^{T} e^{-\int_{0}^{t} \beta(s) d s} u^{\prime}\left(c^{*}(t)\right)\left(c^{*}(t)-c(t)\right) d t+e^{-\int_{0}^{T} \beta(s) d s} u^{\prime}\left(X^{*}(T)\right)\left(X^{*}(T)-X(T)\right)\right]=0
\end{aligned}
$$

for any feasible strategy, $(c, X)$, with $X(0)=x_{0}$.

We are now ready to prove that the put option-based strategy defined in Proposition 2 is optimal among all feasible strategies, which satisfies the European capital guarantee.

Proof of optimality. Let $(c, \pi) \in \mathcal{A}^{\prime}$, with corresponding wealth process, $(X(t))_{t \in[0, T]}$, be any given feasible strategy satisfying $X(0)=x_{0}$ and $X(T) \geq K$. Using the concavity of $u$, we get that:

$$
\begin{aligned}
& \int_{0}^{T} e^{-\int_{0}^{t} \beta(s) d s} u(c(t)) d t+e^{-\int_{0}^{T} \beta(s) d s} u(X(T)) \\
& -\left(\int_{0}^{T} e^{-\int_{0}^{t} \beta(s) d s} u(\widehat{c}(t)) d t+e^{-\int_{0}^{T} \beta(s) d s} u\left(\widehat{X}^{(\lambda)}(T)\right)\right) \\
= & \int_{0}^{T} e^{-\int_{0}^{t} \beta(s) d s}(u(c(t))-u(\widehat{c}(t))) d t+e^{-\int_{0}^{T} \beta(s) d s}\left(u(X(T))-u\left(\widehat{X}^{(\lambda)}(T)\right)\right) \\
\leq & \int_{0}^{T} e^{-\int_{0}^{t} \beta(s) d s} u^{\prime}(\widehat{c}(t))(c(t)-\widehat{c}(t)) d t+e^{-\int_{0}^{T} \beta(s) d s} u^{\prime}\left(\widehat{X}^{(\lambda)}(T)\right)\left(X(T)-\widehat{X}^{(\lambda)}(T)\right) \\
:= & (*)
\end{aligned}
$$

Since $(c, \pi)$ was arbitrarily chosen, we end the proof by showing that $E[(*)] \leq 0$.

A special feature of the CRRA utility is that $u^{\prime}(x y)=u^{\prime}(x) u^{\prime}(y)$. Using this feature, we get:

$$
u^{\prime}(\widehat{c}(t))(c(t)-\widehat{c}(t))=u^{\prime}(\lambda) u^{\prime}\left(c^{*}(t)\right)\left(c(t)-c^{*}(t)\right)+u^{\prime}(\lambda) u^{\prime}\left(c^{*}(t)\right)\left(c^{*}(t)-\widehat{c}(t)\right)
$$


By use of (23) and noting that $u^{\prime}$ is a decreasing function, we obtain:

$$
\begin{aligned}
& u^{\prime}\left(\widehat{X}^{(\lambda)}(T)\right)\left(X(T)-\widehat{X}^{(\lambda)}(T)\right) \\
= & \left(u^{\prime}(\lambda) u^{\prime}\left(X^{*}(T)\right) \wedge u^{\prime}(K)\right)\left(X(T)-\widehat{X}^{(\lambda)}(T)\right) \\
= & u^{\prime}(\lambda) u^{\prime}\left(X^{*}(T)\right)\left(X(T)-\widehat{X}^{(\lambda)}(T)\right)-\left(u^{\prime}(\lambda) u^{\prime}\left(X^{*}(T)\right)-u^{\prime}(K)\right)^{+}(X(T)-K)
\end{aligned}
$$

where we, in the last equality, have used that $\widehat{X}^{(\lambda)}(T)=K$ on the set $\left\{(T, \omega): u^{\prime}(\lambda) u^{\prime}\left(X^{*}(T)\right) \geq u^{\prime}(K)\right\}$. Since, by assumption, $X(T) \geq K$, this reduces to:

$$
\begin{aligned}
& u^{\prime}\left(\widehat{X}^{(\lambda)}(T)\right)\left(X(T)-\widehat{X}^{(\lambda)}(T)\right) \\
\leq & u^{\prime}(\lambda) u^{\prime}\left(X^{*}(T)\right)\left(X(T)-\widehat{X}^{(\lambda)}(T)\right) \\
= & u^{\prime}(\lambda) u^{\prime}\left(X^{*}(T)\right)\left(X(T)-X^{*}(T)\right)+u^{\prime}(\lambda) u^{\prime}\left(X^{*}(T)\right)\left(X^{*}(T)-\widehat{X}^{(\lambda)}(T)\right)
\end{aligned}
$$

Finally, combining (24) and (25) and using Lemma 3 twice give us $E[(*)] \leq 0$.

\section{The American Capital Guarantee Control Problem}

Consider the problem:

$$
\sup _{(c, \pi) \in \mathcal{A}^{\prime}} E\left[\int_{0}^{T} e^{-\int_{0}^{t} \beta(s) d s} u(c(t)) d t+e^{-\int_{0}^{T} \beta(s) d s} u(X(T))\right]
$$

under the capital constraint, $X(t) \geq K(t), \forall t \in[0, T]$, where $K$ is a deterministic function of time. In Section 3, we saw that $K(t) \leq-g(t), \forall t \in[0, T]$, is a non-binding constraint. Note that $K=0$ corresponds to the case where the investor is restricted from borrowing against future labor income. In order for the American capital guarantee problem to be solvable and non-trivial, ${ }^{6}$ we must demand:

$$
K(t)<e^{r t}\left(x_{0}+\int_{0}^{t} e^{-r s} \ell(s) d s\right), \forall t \in(0, T]
$$

Remark 5 presents the case without terminal consumption. Equivalent to the unrestricted problem, we get that the optimal strategies in the cases with and without terminal consumption are very closely related.

\subsection{An Admissible American Put Option-Based Portfolio}

In Subsection 5.2, we present the optimal strategy for Problem (26). In turns out that the optimal strategy is very similar to the optimal strategy for the European capital guarantee problem presented in Proposition 2. Again, the optimal strategy consists of a position in a budget-adjusted version of the optimal portfolio derived for the unrestricted problem plus a put option written on that portfolio. This

6 In the case of equality in (27), the investor has no choice, but to invest all his wealth, including labor income, in the risk-free short rate to be sure to fulfil the capital guarantee. 
time though, to fulfil the American capital guarantee, the OBPI strategy must consist of an American put option. However, the introduction of an American put option is not uncomplicated. It turns out that the part of the strategy that consists of the American put option is not admissible by default. Loosely speaking, to make the strategy admissible, we have to continuously adjust the budget whenever the constraint is active. In the following, this will be made clear.

Still, we denote by $X^{*}(t), c^{*}(t)$ and $\pi^{*}(t), t \in[0, T]$, the optimal wealth, the optimal consumption strategy and the optimal investment strategy for the unrestricted problem (8), respectively. Observe that the portfolio $Y^{*}(t)=X^{*}(t)+g(t), t \in[0, T]$, has dynamics:

$$
\begin{aligned}
d Y^{*}(t) & =\left(r+\frac{1}{\gamma}\left(\frac{\alpha-r}{\sigma}\right)^{2}-\frac{1}{f(t)}\right) Y^{*}(t) d t+\frac{1}{\gamma} \frac{\alpha-r}{\sigma} Y^{*}(t) d W(t) \\
& =\left(r-\frac{1}{f(t)}\right) Y^{*}(t) d t+\frac{1}{\gamma} \frac{\alpha-r}{\sigma} Y^{*}(t) d W^{Q}(t), t \in[0, T) \\
Y^{*}(0) & =y_{0}
\end{aligned}
$$

where $y_{0}=x_{0}+g(0)$. Denote by $P_{y}^{a}(t, T, K+g)$ the time, $t$, value of an American put option with strike price, $K(s)+g(s), \forall s \in[t, T]$, and maturity $T$ written on a portfolio, $Y$, where $(Y(s))_{s \in[t, T]}$ is the solution to (28), which equals $y$ at time $t$. By definition, the price of such a put option is given by:

$$
P_{y}^{a}(t, T, K+g):=\sup _{\tau \in \mathcal{T}_{t, T}} E^{Q}\left[e^{-r(\tau-t)}(K(\tau)+g(\tau)-Y(\tau))^{+} \mid Y(t)=y\right], \forall t \in[0, T]
$$

where $\mathcal{T}_{t, T}$ is the set of stopping times taking values in the interval $[t, T]$.

By analogy with the European case, we introduce the American put option-based portfolio:

$$
X^{(\lambda)}(t):=\lambda Y^{*}(t)+P_{\lambda Y^{*}}^{a}(t, T, K+g)-g(t), \forall t \in[0, T]
$$

where $\lambda \in(0,1)$ is determined by the budget constraint:

$$
X^{(\lambda)}(0)=\lambda\left(x_{0}+g(0)\right)+P_{\lambda y_{0}}^{a}(0, T, K+g)-g(0)=x_{0}
$$

By definition of an American put option $P_{\lambda Y^{*}}^{a}(t, T, K+g) \geq\left(K(t)+g(t)-\lambda Y^{*}(t)\right)^{+}, \forall t \in[0, T]$. We conclude that $X^{(\lambda)}$ fulfills the American capital guarantee, since, $\forall t \in[0, T]$, we have:

$$
\begin{aligned}
X^{(\lambda)}(t) & =\lambda Y^{*}(t)+P_{\lambda Y^{*}}^{a}(t, T, K+g)-g(t) \\
& \geq \lambda Y^{*}(t)+\left(K(t)+g(t)-\lambda Y^{*}(t)\right)^{+}-g(t) \\
& \geq K(t)
\end{aligned}
$$

Now, recall some basis properties for American put options in a Black-Scholes market (see, e.g., Karatzas and Shreve [14]):

$$
\begin{aligned}
P_{y}^{a}(t, T, K+g) & =K(t)+g(t)-y, & \forall(t, y) & \in \mathcal{C}^{c} \\
\mathcal{L} P_{y}^{a}(t, T, K+g) & =r P_{y}^{a}(t, T, K+g), & \forall(t, y) & \in \mathcal{C} \\
\frac{\partial}{\partial y} P_{y}^{a}(t, T, K+g) & =-1, & \forall(t, y) & \in \mathcal{C}^{c}
\end{aligned}
$$


where (see (28) and (19)):

$$
\mathcal{L}:=\frac{\partial}{\partial t}+\left(r-\frac{1}{f(t)}\right) y \frac{\partial}{\partial y}+\frac{1}{2} \sigma^{2}\left(\pi_{y}^{*}\right)^{2} y^{2} \frac{\partial^{2}}{\partial y^{2}}
$$

and:

$$
\mathcal{C}:=\left\{(t, y): P_{y}^{a}(t, T, K+g)>(K(t)+g(t)-y)^{+}\right\}
$$

defines the continuation region. By $\mathcal{C}^{c}$, we mean the complementary of $\mathcal{C}$, i.e., the stopping region. The continuation region can be described via the exercise boundary:

$$
b(t)=\sup \left\{y: P_{y}^{a}(t, T, K+g)=(K(t)+g(t)-y)^{+}\right\}
$$

Note that $b$ is a deterministic function of time. We get:

$$
\mathcal{C}=\{(t, y): y>b(t)\}
$$

Introducing the function, $A$, by:

$$
A(t, y):=y+P_{y}^{a}(t, T, K+g)-g(t)
$$

we can write (29) as:

$$
X^{(\lambda)}(t)=A\left(t, \lambda Y^{*}(t)\right), \forall t \in[0, T]
$$

From the properties of $P_{y}^{a}(t, T, K+g)$, we deduce that:

$$
\begin{array}{rlrl}
A(t, y) & =K(t), & & \forall(t, y) \in \mathcal{C}^{c}, \\
\mathcal{L} A(t, y) & =\left(r-\frac{1}{f(t)}\right) y+r P_{y}^{a}(t, T, K+g)-(-\ell(t)+r g(t)) & & \\
& =r A(t, y)+\ell(t)-\frac{y}{f(t)}, & & \forall(t, y) \in \mathcal{C} \\
\mathcal{L} A(t, y) & =\frac{\partial}{\partial t} K(t), & \forall(t, y) \in \mathcal{C}^{c} \\
\frac{\partial}{\partial y} A(t, y) & =0 . & \forall(t, y) \in \mathcal{C}^{c}
\end{array}
$$

Since $Y^{*}(t)$ is linear in its initial value, $\forall t \in[0, T]$, we have that $\lambda Y^{*}(t)$ has the same dynamics as $Y^{*}(t)$, $\forall t \in[0, T]$. By use of (32), (33) and (18), we get that: ${ }^{7}$

$$
\begin{aligned}
d A\left(t, \lambda Y^{*}(t)\right)= & \frac{\partial}{\partial y} A\left(t, \lambda Y^{*}(t)\right) \sigma \pi_{y}^{*} \lambda Y^{*}(t) d W^{Q}(t)+\mathcal{L} A\left(t, \lambda Y^{*}(t)\right) d t \\
= & \frac{\partial}{\partial y} A\left(t, \lambda Y^{*}(t)\right) \sigma \pi_{y}^{*} \lambda Y^{*}(t) d W^{Q}(t) \\
& +\left[r A\left(t, \lambda Y^{*}(t)\right)+\ell(t)-\lambda c^{*}(t)\right] 1_{\left(\lambda Y^{*}(t)>b(t)\right)} d t+\frac{\partial}{\partial t} K(t) 1_{\left(\lambda Y^{*}(t) \leq b(t)\right)} d t
\end{aligned}
$$

From Condition (7), we see that the strategy, $\left(\lambda c^{*}, X^{(\lambda)}\right)$, is admissible up to the hitting time of the exercise boundary, $b$. If we allow $\lambda$ to be a function of time, we can choose $(\lambda(t), t \in[0, T])$ to be

$7 \frac{\partial}{\partial y}$ now means differentiating w.r.t. the second variable. 
constant whenever $\lambda(t) Y^{*}(t)>b(t)$ and increasing at the boundary, such that $\lambda(t) Y^{*}(t) \geq b(t), \forall t \in$ $[0, T]$. More precisely, define:

$$
\lambda(t)=\lambda_{0} \vee \sup _{s \leq t}\left(\frac{b(s)}{Y^{*}(s)}\right), \forall t \in[0, T]
$$

where $\lambda_{0}$ is determined by the budget constraint (30).

Proposition 4. The strategy $\left(\lambda c^{*}, X^{(\lambda)}\right)$, where, $\forall t \in[0, T]$,

$$
X^{(\lambda)}(t):=\lambda(t) Y^{*}(t)+P_{\lambda Y^{*}}^{a}(t, T, K)-g(t)
$$

with $\lambda(t), t \in[0, T]$, being the function defined by (36) and (30), is admissible ${ }^{8}$.

Proof. Using Itö's formula, (35) and that $\lambda$ increases only at the boundary, we get that:

$$
\begin{aligned}
d A\left(t, \lambda(t) Y^{*}(t)\right)= & {\left[d A\left(t, \lambda Y^{*}(t)\right)\right]_{\mid \lambda=\lambda(t)}+Y^{*}(t) \frac{\partial}{\partial y} A\left(t, \lambda(t) Y^{*}(t)\right) d \lambda(t) } \\
= & \frac{\partial}{\partial y} A\left(t, \lambda(t) Y^{*}(t)\right) \sigma \pi_{y}^{*} \lambda(t) Y^{*}(t) d W^{Q}(t) \\
& +\left[r A\left(t, \lambda(t) Y^{*}(t)\right)+\ell(t)-\lambda(t) c^{*}(t)\right] 1_{\left(\lambda(t) Y^{*}(t)>b(t)\right)} d t+\frac{\partial}{\partial t} K(t) 1_{\left(\lambda(t) Y^{*}(t) \leq b(t)\right)} d t \\
& +Y^{*}(t) \frac{\partial}{\partial y} A\left(t, \lambda(t) Y^{*}(t)\right) 1_{\left(\lambda(t) Y^{*}(t)=b(t)\right)} d \lambda(t)
\end{aligned}
$$

Since by (34) $\frac{\partial}{\partial y} A\left(t, \lambda(t) Y^{*}(t)\right)=0$ on the set $\left\{(t, \omega): \lambda(t) Y^{*}(t)=b(t)\right\}$ this reduces to

$$
\begin{aligned}
d A\left(t, \lambda(t) Y^{*}(t)\right)= & \frac{\partial}{\partial y} A\left(t, \lambda(t) Y^{*}(t)\right) \sigma \pi_{y}^{*} \lambda(t) Y^{*}(t) d W^{Q}(t)+\left[r A\left(t, \lambda(t) Y^{*}(t)\right)+\ell(t)-\lambda(t) c^{*}(t)\right] d t \\
& +\left(\frac{\partial}{\partial t} K(t)-\left(r K(t)+\ell(t)-\lambda(t) c^{*}(t)\right)\right) 1_{\left(\lambda(t) Y^{*}(t) \leq b(t)\right)} d t
\end{aligned}
$$

Finally, since $\left\{(t, \omega): \lambda(t) Y^{*}(t) \leq b(t)\right\}=\left\{(t, \omega): \lambda(t)=\frac{b(t)}{Y^{*}(t)}\right\}$ has a zero $d t \otimes d P$ measure, we conclude that:

$d A\left(t, \lambda(t) Y^{*}(t)\right)=\left[r A\left(t, \lambda(t) Y^{*}(t)\right)+\ell(t)-\lambda(t) c^{*}(t)\right] d t+\frac{\partial}{\partial y} A\left(t, \lambda(t) Y^{*}(t)\right) \sigma \pi_{y}^{*} \lambda(t) Y^{*}(t) d W^{Q}(t)$ i.e., by (7), the strategy is admissible.

\subsection{The Optimal Strategy}

As previously mentioned, the put option-based strategy introduced in Subsection 5.1 turns out to be optimal for the American capital guarantee problem (26).

8 It is implicit, given that $P_{\lambda Y^{*}}^{a}(t, T, K)$ now refers to an American put option written on the portfolio, $\left(\lambda(s) Y^{*}(s)\right)_{s \in[t, T]}$. 
Theorem 5. Consider the strategy $\left(\widehat{c}, \lambda Y^{*}\right)$, where, $\forall t \in[0, T]$,

$$
\begin{aligned}
\widehat{c}(t) & :=\frac{\lambda(t) Y^{*}(t)}{f(t)}=\lambda(t) c^{*}(t) \\
Y^{*}(t) & :=X^{*}(t)+g(t)
\end{aligned}
$$

combined with a position in an American put option with strike price $K(s)+g(s), \forall s \in[t, T]$, and maturity $T$ written on the portfolio $\left(\lambda(s) Y^{*}(s)\right)_{s \in[t, T]}$, where $\lambda(s), s \in[t, T]$, is the function defined by (36) and (30). By (31), the value of the corresponding option based portfolio insurance becomes:

$$
\widehat{X}^{(\lambda)}(t):=\lambda(t) Y^{*}(t)+P_{\lambda Y^{*}}^{a}(t, T, K+g)-g(t) \geq K(t)
$$

i.e., the American capital guarantee is fulfilled. Observing that $Y^{*}(T)=X^{*}(T)$, the terminal value becomes:

$$
\widehat{X}^{(\lambda)}(T)=\lambda(T) X^{*}(T)+\left(K(T)-\lambda(T) X^{*}(T)\right)^{+}=\max \left(\lambda(T) X^{*}(T), K(T)\right)
$$

We have that:

- The strategy is optimal for the American capital guarantee control problem given by (26).

Note that, clearly, the strategy in Theorem 5 is feasible since the unrestricted strategy $\left(c^{*}, \pi^{*}\right)$ given in Section 3 is feasible.

Remark 4. To clarify, following the strategy $\left(\widehat{c}, \widehat{X}^{(\lambda)}\right)$ given by Theorem 5 (heuristically) means that the investor should:

(1) Take a loan of size $g(0)$ and reserve the labor income to pay back the loan over the time interval $[0, T]$.

(2) Reserve the initial amount of money $\lambda_{0}\left(x_{0}+g(0)\right)$ to follow the consumption and investment strategy $(\widehat{c}, \pi)$ given by:

$$
\begin{aligned}
\widehat{c}(t) & :=\lambda_{0} c^{*}(t) \\
\pi & :=\pi_{y}^{*}
\end{aligned}
$$

Doing this, the investor replicates the portfolio $\lambda_{0} Y^{*}(t), \forall t \in[0, T]$ (see Remark 2).

(3) Use the remaining initial amount of money $\left(1-\lambda_{0}\right)\left(x_{0}+g(0)\right)$ to buy an American put option with strike price $K(s)+g(s), \forall s \in[0, T]$, and time to maturity $T$ written on the portfolio $\left(\lambda_{0} Y^{*}(t)\right)_{t \in[0, T]}$.

(4) The first time (say $\tau) \lambda_{0} Y^{*}$ drops below the optimal exercise boundary, b, sell the American put option. Not taking the loan we made in (1) into account, the portfolio will then be worth $K(\tau)+$ $g(\tau) .{ }^{9}$ Reserve now the amount of money $\lambda(\tau)(K(\tau)+g(\tau))$ to follow the consumption and investment strategy $(\widehat{c}, \pi)$ given by:

$$
\begin{aligned}
\widehat{c}(t) & :=\lambda(\tau) c^{*}(t) \\
\pi & :=\pi_{y}^{*}
\end{aligned}
$$

9 Taking the loan we made in (1) into account, the portfolio is worth $K(\tau)$, i.e., we stand at the capital guarantee boundary. 
Doing this, the investor replicates the portfolio, $\lambda(\tau) Y^{*}(t), \forall t \in[\tau, T]$. Use the remaining initial amount of money $(1-\lambda(\tau))(K(\tau)+g(\tau))$ to buy an American put option with strike price $K(t)+g(t), \forall t \in[\tau, T]$ and maturity $T$ written on the portfolio, $\lambda(\tau) Y^{*}(t), \forall t \in[\tau, T]$.

(5) In case the new portfolio, $\lambda(\tau) Y^{*}(t)$, drops below the optimal exercise boundary, $b$, at some point in time, $t \in(\tau, T]$, repeat Step 4.

Identically to the European case, the American put option is likely not to be sold in the market, but since the market is complete and frictionless, such options can be replicated dynamically by a Delta-hedge, i.e., the optimal investment strategy, $\widehat{\pi}(t), t \in[0, T]$, is given by:

$$
\widehat{\pi}(t) \widehat{X}^{(\lambda)}(t)=\left(1+\frac{\partial}{\partial y} P_{\lambda Y^{*}}^{a}(t, T, K+g)\right) \pi_{y}^{*} \lambda(t) Y^{*}(t)
$$

One should notice that at the boundary, no risk is taken, i.e., the position in the American put option offsets the position in the underlying portfolio, such that the total exposure to changes in the risky asset, $S$, becomes zero. Clearly, at the boundary, it is optimal to consume a certain time-dependent part of the labor income, thereby leaving room for a risky position in $S$ immediately after hitting the capital boundary. This is numerically illustrated in Subsection 5.3.

Proof of Optimality. Let $(c, \pi) \in \mathcal{A}^{\prime}$, with corresponding wealth process, $(X(t))_{t \in[0, T]}$, be any given feasible strategy satisfying $X(0)=x_{0}$ and $X(t) \geq K(t), \forall t \in[0, T]$. Since $u$ is concave, we get:

$$
\begin{aligned}
& \int_{0}^{T} e^{-\int_{0}^{t} \beta(s) d s} u(c(t)) d t+e^{-\int_{0}^{T} \beta(s) d s} u(X(T)) \\
& -\left(\int_{0}^{T} e^{-\int_{0}^{t} \beta(s) d s} u(\widehat{c}(t)) d t+e^{-\int_{0}^{T} \beta(s) d s} u\left(\widehat{X}^{(\lambda)}(T)\right)\right) \\
= & \int_{0}^{T} e^{-\int_{0}^{t} \beta(s) d s}(u(c(t))-u(\widehat{c}(t))) d t+e^{-\int_{0}^{T} \beta(s) d s}\left(u(X(T))-u\left(\widehat{X}^{(\lambda)}(T)\right)\right) \\
\leq & \int_{0}^{T} e^{-\int_{0}^{t} \beta(s) d s} u^{\prime}(\widehat{c}(t))(c(t)-\widehat{c}(t)) d t+e^{-\int_{0}^{T} \beta(s) d s} u^{\prime}\left(\widehat{X}^{(\lambda)}(T)\right)\left(X(T)-\widehat{X}^{(\lambda)}(T)\right) \\
= & :(*)
\end{aligned}
$$

Since $(c, \pi)$ was arbitrarily chosen, we end the proof by showing that $E[(*)] \leq 0$.

Again, by the CRRA property $u^{\prime}(x y)=u^{\prime}(x) u^{\prime}(y)$, we have:

$$
u^{\prime}(\widehat{c}(t))(c(t)-\widehat{c}(t))=u^{\prime}(\lambda(t)) u^{\prime}\left(c^{*}(t)\right)(c(t)-\widehat{c}(t))
$$

By use of (38) and noting that $u^{\prime}$ is a decreasing function, we get that:

$$
\begin{aligned}
& u^{\prime}\left(\widehat{X}^{(\lambda)}(T)\right)\left(X(T)-\widehat{X}^{(\lambda)}(T)\right) \\
= & \left(u^{\prime}(\lambda(T)) u^{\prime}\left(X^{*}(T)\right) \wedge u^{\prime}(K(T))\right)\left(X(T)-\widehat{X}^{(\lambda)}(T)\right) \\
= & u^{\prime}(\lambda(T)) u^{\prime}\left(X^{*}(T)\right)\left(X(T)-\widehat{X}^{(\lambda)}(T)\right)-\left(u^{\prime}(\lambda(T)) u^{\prime}\left(X^{*}(T)\right)-u^{\prime}(K(T))\right)^{+}(X(T)-K(T))
\end{aligned}
$$

where the last equality is established by using that $\widehat{X}^{(\lambda)}(T)=K(T)$ on the set $\left\{(T, \omega): u^{\prime}\left(\lambda(T) X^{*}(T)\right) \geq u^{\prime}(K(T))\right\}$. Since, by assumption, $X(t) \geq K(t), \forall t \in[0, T]$, this reduces to:

$$
u^{\prime}\left(\widehat{X}^{(\lambda)}(T)\right)\left(X(T)-\widehat{X}^{(\lambda)}(T)\right) \leq u^{\prime}(\lambda(T)) u^{\prime}\left(X^{*}(T)\right)\left(X(T)-\widehat{X}^{(\lambda)}(T)\right)
$$


Inserting (41) and (42) and then (12) and (13), we get:

$$
\begin{aligned}
E[(*)]:= & E\left[\int_{0}^{T} e^{-\int_{0}^{t} \beta(s) d s} u^{\prime}(\widehat{c}(t))(c(t)-\widehat{c}(t)) d t\right]+E\left[e^{-\int_{0}^{T} \beta(s) d s} u^{\prime}\left(\widehat{X}^{(\lambda)}(T)\right)\left(X(T)-\widehat{X}^{(\lambda)}(T)\right)\right] \\
\leq & E\left[\int_{0}^{T} e^{-\int_{0}^{t} \beta(s) d s} u^{\prime}(\lambda(t)) u^{\prime}\left(c^{*}(t)\right)(c(t)-\widehat{c}(t)) d t\right] \\
& +E\left[e^{-\int_{0}^{T} \beta(s) d s} u^{\prime}(\lambda(T)) u^{\prime}\left(X^{*}(T)\right)\left(X(T)-\widehat{X}^{(\lambda)}(T)\right)\right] \\
= & E\left[\int_{0}^{T} e^{-\int_{0}^{t} \beta(s) d s} u^{\prime}(\lambda(t)) \xi^{*} H(t)(c(t)-\widehat{c}(t)) d t\right] \\
& +\left[e^{-\int_{0}^{T} \beta(s) d s} u^{\prime}(\lambda(T)) \xi^{*} H(T)\left(X(T)-\widehat{X}^{(\lambda)}(T)\right)\right] \\
= & \xi^{*} E^{Q}\left[\int_{0}^{T} e^{-r t} u^{\prime}(\lambda(t))(c(t)-\widehat{c}(t)) d t+e^{-r T} u^{\prime}(\lambda(T))\left(X(T)-\widehat{X}^{(\lambda)}(T)\right)\right]
\end{aligned}
$$

Since $u^{\prime}(\lambda(t))$ is a decreasing function, ${ }^{10}$ we can use the integration by parts formula:

$$
\begin{aligned}
E[(*)] \leq & \xi^{*}\left(E^{Q}[\underbrace{\int_{0}^{T} e^{-r t} u^{\prime}(\lambda(t))(c(t)-\widehat{c}(t)) d t+\int_{0}^{T} u^{\prime}(\lambda(t)) d\left(e^{-r t}\left(X(t)-\widehat{X}^{(\lambda)}(t)\right)\right)}_{(\star)}]\right. \\
& +E^{Q}[\underbrace{\left.\left.\int_{0}^{T} e^{-r t}\left(X(t)-\widehat{X}^{(\lambda)}(t)\right) d u^{\prime}(\lambda(t))\right]\right)}_{(\star \star)}
\end{aligned}
$$

The second part of (43) can be rewritten as:

$$
\begin{aligned}
E^{Q}[(\star \star)]= & E^{Q}\left[\int_{0}^{T} e^{-r t}(X(t)-K(t)) d u^{\prime}(\lambda(t))\right] \\
& +E^{Q}\left[\int_{0}^{T} e^{-r t}\left(K(t)-\widehat{X}^{(\lambda)}(t)\right) d u^{\prime}(\lambda(t))\right]
\end{aligned}
$$

The first term is non-positive, since per definition $X(t) \geq K(t), \forall t \in[0, T]$, and $d u^{\prime}(\lambda(t)) \leq 0$, $\forall t \in[0, T]$ ( $u^{\prime}$ is decreasing and $\lambda$ is increasing). The second term equals zero, since $d u^{\prime}(\lambda(t)) \neq 0$ only on the set $\left\{(t, \omega): \widehat{X}^{(\lambda)}(t)=K(t)\right\}$. We conclude that $E^{Q}[(\star \star)] \leq 0$. The first part of (43) can be rewritten as:

$$
\begin{aligned}
E^{Q}[(\star)]= & E^{Q}\left[\int_{0}^{T} u^{\prime}(\lambda(t)) d\left(\int_{0}^{t} e^{-r s}(c(s)-\widehat{c}(s)) d s\right)\right] \\
& +E^{Q}\left[\int_{0}^{T} u^{\prime}(\lambda(t)) d\left(e^{-r t}\left(X(t)-\widehat{X}^{(\lambda)}(t)\right)\right)\right] \\
= & E^{Q}\left[\int_{0}^{T} u^{\prime}(\lambda(t)) d\left(\int_{0}^{t} e^{-r s}(c(s)-\ell(s)) d s+e^{-r t} X(t)\right)\right] \\
& -E^{Q}\left[\int_{0}^{T} u^{\prime}(\lambda(t)) d\left(\int_{0}^{t} e^{-r s}(\widehat{c}(s)-\ell(s)) d s+e^{-r t} \widehat{X}^{(\lambda)}(t)\right)\right]
\end{aligned}
$$

10 This ensures that the stochastic integral in (43) is well-defined. 
Since both strategies are admissible, we note that, according to (6), Equation (44) consists of two stochastic integrals with respect to $Q$-martingales. Since $u^{\prime}(\lambda(t)) \leq u^{\prime}\left(\lambda_{0}\right), \forall t \in[0, T]$, we get:

$$
E^{Q}[(\star)]=0
$$

Finally, we can conclude that:

$$
E[(*)]=E^{Q}[(\star)]+E^{Q}[(\star \star)] \leq 0
$$

Remark 5. As a final remark, we want to emphasize that the case where the investor assigns zero utility to terminal wealth can be handled equivalently as in Sections 3-5. To be clear, consider the unrestricted problem:

$$
\sup _{(c, \pi) \in \mathcal{A}^{\prime}} E\left[\int_{0}^{T} e^{-\int_{0}^{t} \beta(s) d s} u(c(t)) d t\right]
$$

where the investor's wealth follows the dynamics given by (1) with initial wealth equal to $x_{0}$. It is well known that the solution to (45) is equivalent to the unrestricted solution given by (14) and (15), the only difference being that the deterministic function, $f$, is now given by:

$$
f(t)=\int_{t}^{T} e^{-\int_{t}^{s}[\widetilde{r}(y)+\beta(y)] d y} d s
$$

with $\widetilde{r}(y)=\frac{\gamma}{\gamma} \beta(y)-\frac{\gamma}{\gamma} r-\frac{1}{2} \frac{\gamma}{(1-\gamma)^{2}}\left(\frac{\alpha-r}{\sigma}\right)^{2}$. Now $f$ converges to zero as time goes to $T$. Consequently, since by (14)

$$
c^{*}(t)=\frac{X(t)+g(t)}{f(t)}
$$

we notice that the optimal terminal wealth will be zero (since the investor assigns no utility to the terminal wealth, he simply consumes everything before time T). The solution to (45) under a European or an American capital guarantee is equivalent to the solutions presented in Proposition 2, respectively, Theorem 5, the only difference being that $f$ is now given by (46).

\subsection{Numerical Illustrations}

In this subsection, we illustrate the optimal strategy presented in Theorem 5 in the case with a restriction to borrow against future labor income, i.e., $K(t)=0, \forall t \in[0, T]$. Naturally, we compare the strategy to the unrestricted strategy derived in Section 3. More specifically, consider an investor with time horizon $T=10$, time and utility preferences $(\beta, \gamma)=(0.01,-2)$, endowed with zero initial wealth and a labor income rate starting out at $\ell(0)=40,000$ followed by a monthly half a percent raise. The investor faces a Black-Scholes market with parameters $(\alpha, r, \sigma)=(0.12,0.04,0.2)$ resulting in $\pi_{y}^{*}=\frac{1}{\gamma} \frac{\alpha-r}{\sigma^{2}}=\frac{2}{3}$. The time interval has been discretized equidistantly into 120 time points, i.e., monthly time points. By this, we mean that the American put option has been evaluated as a discrete time option, such that the guarantee is only valid monthly. Likewise, $\lambda$ and, therefore, the optimal consumption and investment strategy, has only been changed monthly. Saying that, it is important to stress that in-between 
the monthly time points, the amount of money to consume and invest, respectively, has been re-balanced continuously. ${ }^{11}$

Figure 1. (Upper left) The underlying portfolio, $\lambda(t) Y^{*}(t)$ (solid curve), together with the strike price, $g(t)$ (dotted curve), and the optimal exercise boundary, $b(t)$, of the American put option (dashed curve). (Upper right) $\lambda(t)$. (Lower left) The American put option (solid curve) together with the optimal exercise value (dashed curve). (Lower right) The optimal portfolio, $\widehat{X}^{(\lambda)}(t)$ (solid curve), together with the corresponding optimal unrestricted portfolio, $X^{*}(t)$ (dashed curve), and the guarantee $(K=0)$.
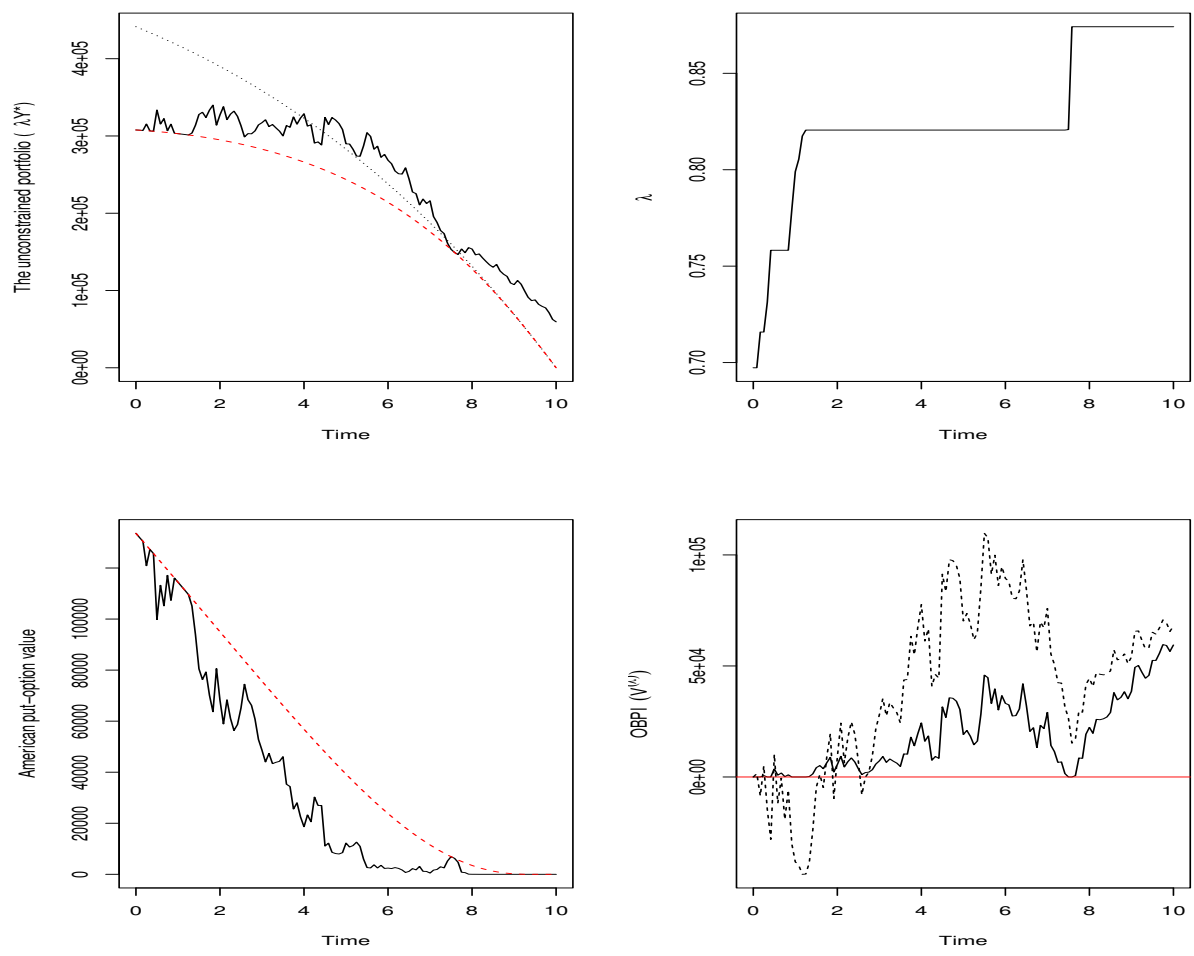

Figure 1 illustrates the optimal portfolio together with its building blocks (the underlying portfolio and the associated American put option). The investor starts out by splitting the initial borrowed amount of money $g(0)=441,361.8$ into two portions: one of size $\lambda(0) g(0)=307,759.9$, reserved to finance the investment and consumption strategy given by (39) and (40) (i.e., a hedge of $\lambda_{0} Y^{*}$ ), and one of size $(1-\lambda(0)) g(0)=133,601.9$, used to buy (or delta-hedge) an American put option with strike $g(t)$ and maturity $T$ written on the underlying portfolio, $\lambda_{0} Y^{*}$. Looking at Figure 1 (upper left plot), we see that initially, this corresponds to choosing an underlying portfolio of a size equal to the exercise boundary of the corresponding American put option. One can realize that this is always the case when the initial amount of money equals the capital guarantee, $K(0)$.

As seen in Figure 1, as time goes by, the optimal portfolio hits the boundary. For this specific example, we observe (after evaluating a large number of sample paths) that the optimal portfolio hits the boundary

11 We have used (16). Note that $X(t)^{*}+g(t), t \in[0, T]$, is a geometric Brownian motion, since we have chosen $\beta$ to be constant. 
within the (say) first three years, with a rather large probability, and that it hits the boundary in the (say) last two years before terminal time $T$ with a negligible probability. Realizations like this can often be found to be a consequence of the model design. In this example, the reason is partly due to the fact that the investor is endowed with an increasing labor income and no initial wealth. Looking at the optimal consumption strategy (39), one observes that this labor income design causes the investor, who holds a small portfolio, to save a little fraction of the labor income in the beginning of the period, whereas he chooses to save a large fraction of the labor income in the years just before terminal time $T$ (see Figure 2).

Figure 2. The optimal consumption rate (solid curve) together with the optimal unrestricted consumption rate (dashed curve), the deterministic labor income rate (dotted curve) and the deterministic optimal boundary consumption rate (solid smooth curve).

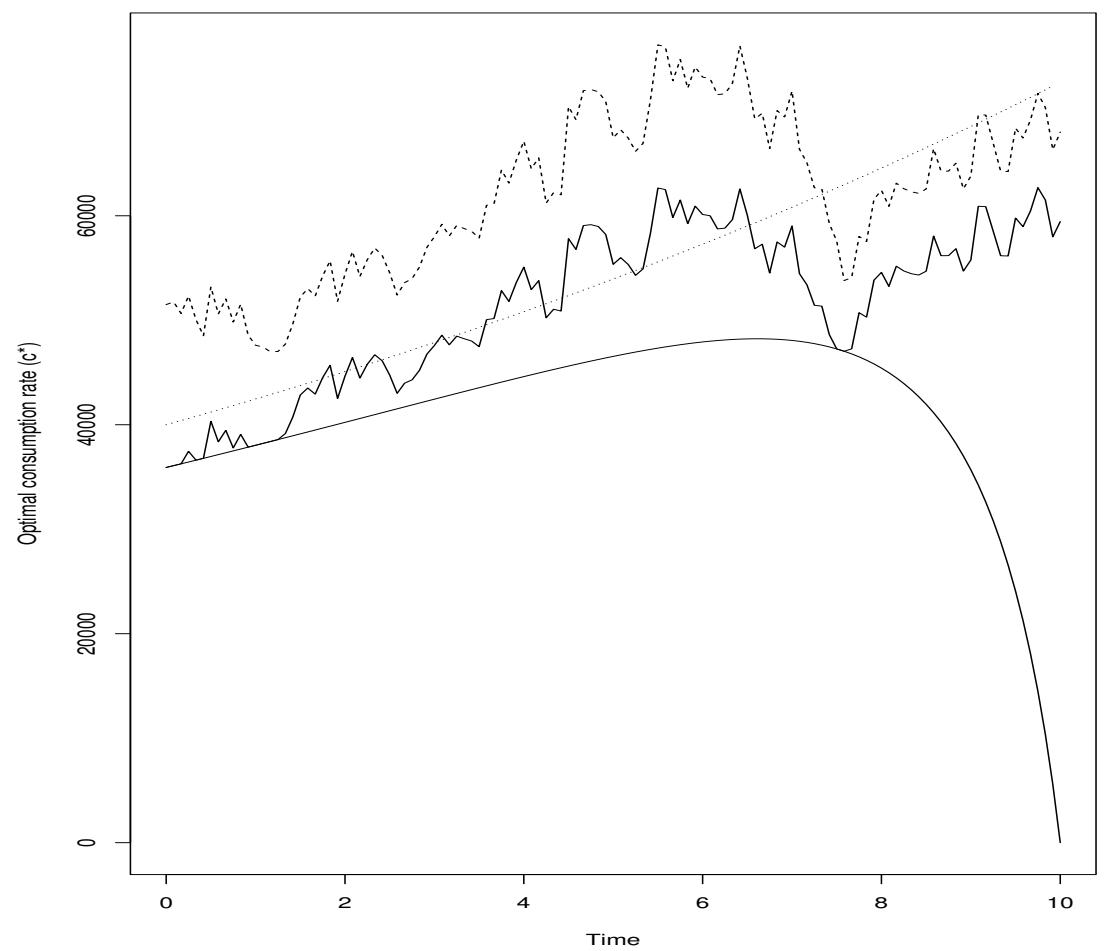

In Section 5.1, we found how to change $\lambda$ dynamically in order for the put option-based strategy to be admissible. In Figure 1 (upper right plot), we see how $\lambda(t), t \in[0, T]$, increases when the underlying process, $\left(\lambda Y^{*}\right)_{t \in[0, T]}$, hits the exercise boundary of the American put option. Consequently, the American put option never exceeds its inner value, as seen in Figure 1 (lower left plot).

Finally, Figure 1 (lower right plot) shows the optimal portfolio plotted against the corresponding unrestricted optimal portfolio. We see how the optimal portfolio stays above or at zero during the time period, while the unrestricted optimal portfolio becomes negative over several distinct time intervals. The fact that the optimal portfolio increases drastically away from zero the last two years before terminal time $T$ is primary due to the fact that the investor's optimal consumption strategy leaves room for saving labor income in the final years whenever wealth is sufficiently low (see Figure 2).

In Figure 2, we see how the optimal consumption rate fluctuates around the labor income rate. To begin with, it is optimal to save a certain fraction of the labor income. As investments start to pay off, it 
eventually becomes optimal to consume more than the labor income ( $t \in[2.5,6.5])$. After 6.5 years, the investor loses money on his investments (for this specific sample path), and it therefore becomes optimal for him to start saving money again. One can say that Figure 2 simply shows the investor's preferences for consuming now versus consuming later. Comparing to the optimal unrestricted consumption rate, we see that the restriction to borrow against future labor income has a smoothing effect on consumption (as shown by He and Pages [8]). The reason that the optimal consumption rate at the boundary goes to zero is clear: as time approaches $T$, while wealth is equal to zero, it becomes infinitely valuable to save money, since the marginal utility of terminal wealth goes to infinity as terminal wealth goes to zero.

Figure 3. (Top) The density of the optimal terminal wealth (solid curve) together with the density of the optimal unrestricted terminal wealth (dashed curve). Vertical lines indicate the 95 percent confidence intervals. (Bottom) The optimal fraction of total wealth invested in the risky stock. The solid curve represents the median, while the dashed curves indicate the 95 percent confidence interval. The line $y=\frac{2}{3}$ represents the unrestricted optimal fraction to invest in the risky stock.
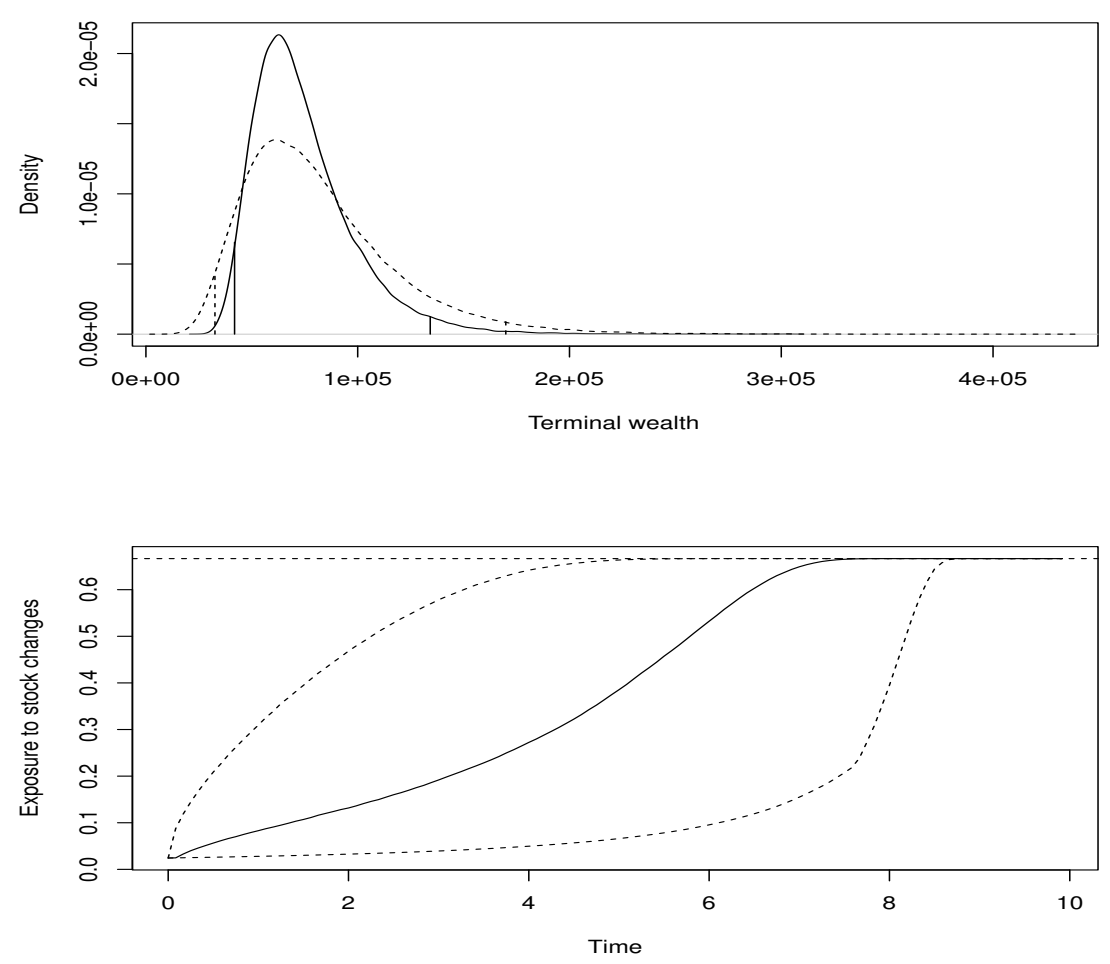

In Figure 3 (upper plot) we compare the optimal terminal wealth with the unrestricted optimal terminal wealth of Merton. Not surprisingly, the restriction to borrow against future labor income results in a much more narrow distribution for the terminal wealth. The optimal terminal wealth has 95 percent of its mass within the interval $[41,854,134,252]$, whereas for the unrestricted problem, this interval is $[32,590,169,928]$. Figure 3 (lower plot) illustrates the risk taken in the optimal portfolio. The risk taken is defined as the fraction of total wealth (wealth plus the financial value of future labor income) invested in the risky stock. We see that the median forms an 's-curve' towards the unrestricted Merton fraction $\left(\pi=\frac{2}{3}\right)$. This is due to the fact that, eventually, the optimal portfolio will increase away from the boundary $(K=0)$ and the American put option will become very cheap, resulting in a strategy 
almost identical to the one of Merton. It should be stressed that we could easily find another example where this will not be the case. The very wide 95 percent confidence interval illustrates how the optimal investment strategy can be very different for different sample paths. It also illustrates how much the optimal investment strategy differs from the unrestricted strategy of Merton. Even in the case where the investor gets a very large and steady payoff from his risky investments, he does not invest nearly as aggressively as Merton before after five years (halfway). Reversely, in the case of very large and steady loses on the investments, the investor places after five years only about 6 percent of total wealth in the risky stock.

\section{Conclusions}

We have solved the classic consumption and investment control problem of Merton in the presence of labor income and an American capital guarantee restriction. The solution obtained is an option-based portfolio insurance (OBPI) combining a long position in the optimal unrestricted allocation with an American put option. More precisely, the investor optimally invests in a budget-adjusted version of the unrestricted portfolio and buys an insurance on that portfolio in the form of an American put option. Initially, the budget for the unrestricted portfolio is adjusted, such that this is affordable. The introduction of consumption and labor income to such a setting has, to the author's knowledge, not been considered before, and the main contribution from this article is to show, by use of the martingale approach to arbitrage-free theory in finance, that the OBPI approach can be carried out to find the optimal strategy in the presence of consumption and labor income. The introduction of a deterministic labor income by itself, in the presence of an American capital guarantee, is not trivial. Unlike the unrestricted problem of Merton, being endowed with a deterministic labor income or being endowed with an initial wealth enlarged by the financial value of future labor income is not equivalent. We have shown that the introduction of deterministic labor income can be handled by choosing an appropriate strike value for the American put option used to insure the investors portfolio from falling below the capital restriction. The strategy, which has to be re-balanced whenever the constraint is active, is rather complicated. Another contribution offered by this article is an in-depth explanation and analysis of the optimal strategy supported by numerical illustrations.

\section{Acknowledgments}

I would like to thank Søren Fiig Jarner (Chief Scientific Officer at ATP, Ph.D.), Mogens Steffensen (Professor at Department of Mathematical Sciences, University of Copenhagen, Ph.D.) and Michael Preisel (Chief Quantitative Officer at ATP, Ph.D.) for helpful comments and discussions during this work.

\section{Conflicts of Interest}

The author declares no conflict of interest. 


\section{References}

1. Merton, R. Lifetime portfolio selection under uncertainty: The continuous-time case. Rev. Econ. Stat. 1969, 51, 247-257.

2. Merton, R. Optimum consumption and portfolio rules in a continuous-time model case. J. Econ. Theory 1971, 3, 373-413.

3. Karatzas, I.; Lehoczky, J.P.; Shreve, S.E. Optimal portfolio and consumption decisions for a 'small investor' on a finite horizon. SIAM J. Control Optim. 1987, 27, 1157-1186.

4. Cox, J.C.; Huang, C.F. Optimal consumption and portfolio policies when asset prices follow a diffusion process. J. Econ. Theory 1989, 49, 33-83.

5. Cox, J.C.; Huang, C.F. A variational problem arising in financial economics. J. Math. Econ. 1991, 20, 465-487.

6. El-Karoui, N.; Jeanblanc, M.; Lacoste, V. Optimal portfolio management with American capital guarantee. J. Econ. Dyn. Control 2005, 29, 449-468.

7. Lakner, P.; Nygren, L.M. Portfolio optimization with downside constraints. Math. Financ. 2006, 2, 283-299.

8. He, H.; Pagès, H.F. Labor income, borrowing constraints, and equilibrium asset prices. Econ. Theory 1993, 3, 663-696.

9. El-Karoui, N.; Jeanblanc, M. Optimization of consumption with labor income. Financ. Stoch. 1998, 2, 409-440.

10. Duffie, D.; Zariphopoulou, T. Optimal investment with undiversifiable income risk. Math. Financ. 1993, 3, 135-148.

11. Duffie, D.; Fleming, W.; Soner, H.; Zariphopoulou, T. Hedging in incomplete markets with HARA utility. J. Econ. Dyn. Control 1997, 21, 753-782.

12. Koo, H. Consumption and portfolio selection with labor income: A continuous time approach. Math. Financ. 1998, 8, 49-65.

13. Munk, C. Optimal consumption/investment policies with undiversifiable income risk and liquidity constraints. J. Econ. Dyn. Control 2000, 24, 1315-1343.

14. Karatzas, I.; Shreve, S.E. Methods of Mathematical Finance; Springer-Verlag: New York, NY, USA, 1998.

15. Fleming, W.H.; Richel, R.W. Deterministic and Stochastic Optimal Control; Springer-Verlag: New York, NY, USA, 1975.

16. Karatzas, I.; Shreve, S.E. Brownian Motion and Stochastic Calculus; Springer-Verlag: New York, NY, USA, 1991.

17. Jensen, B.A.; Sørensen, C. Paying for minimum interest rate guarantees: Who should compensate who? Eur. Financ. Manag. 2001, 25, 183-211.

18. Teplá, L. Optimal investment with minimum performance constraints. J. Econ. Dyn. Control 2001, 25, 1629-1645.

(c) 2014 by the author; licensee MDPI, Basel, Switzerland. This article is an open access article distributed under the terms and conditions of the Creative Commons Attribution license (http://creativecommons.org/licenses/by/3.0/). 